\title{
Full-Wave Analysis of Azimuthally Magnetized Ferrite-Loaded Circular Structures Using the One-Dimensional Finite Difference Frequency Domain Method
}

\author{
Mohammad R. Rawashdeh, Nihad Dib \\ Electrical Engineering Department, Jordan University of Science and Technology, Irbid, Jordan \\ Received 15 September 2008; accepted 26 October 2008
}

\begin{abstract}
Full-wave analysis of circular guiding structures completely filled with ferrite by using the finite difference frequency domain method is presented. The ferrite is assumed to be azimuthally magnetized to remanence. Emphasis is placed on the $\mathrm{TE}_{0 \mathrm{~m}}$ modes that are rotationally symmetric. These modes exhibit nonreciprocal behavior that could be exploited to build phase shifters and microwave isolators. Dispersion diagrams for these modes are given for both forward and reverse waves, and the effect of various ferrite parameters is studied. It is shown that the dispersion diagram may exhibit a region of negative slope, which gives rise to backward wave. (c) 2008 Wiley Periodicals, Inc. Int J RF and Microwave CAE 00: 000-000, 2009.
\end{abstract}

Keywords: finite difference frequency domain; ferrite; isolator; left-hand materials

\section{INTRODUCTION}

Modeling and determining the propagation characteristics of waveguides and transmission lines is a very important issue for the design of microwave circuits. When waveguides and transmission lines are loaded with ferrites, nonreciprocal behavior can be obtained. Ferrites generally have high resistivity and a significant amount of anisotropy at microwave frequencies. The interaction of ferrites with the microwave signal can be controlled by the strength of the DC magnetic bias field. These special properties of ferrites make them suitable for the fabrication of important devices, such as isolators, circulators, switches, resonators, filters, and phase shifters.

Correspondence to: N. Dib; e-mail: nihad@just.edu.jo

DOI $10.1002 /$ mmce. 20362

Published online in Wiley InterScience (www.interscience. wiley.com).
Recently, cylindrical two-dimensional finite difference frequency domain (2D-FDFD) method was used to determine the propagation characteristics of circular guiding structures containing isotropic materials [1-3]. In this article, this technique is extended to analyze coaxial lines and circular waveguides completely filled with azimuthally magnetized ferrite medium. Solving the problems of ferrite loaded waveguides has been a big challenge because of its anisotropic behavior, which appears after applying an external static magnetic field [4-8]. The magnetization properties of ferrite materials are controlled by the external magnetic field $H_{0}$. Ivanov, in $[9,10]$, derived exact wave functions for the wave propagation in azimuthally magnetized ferrite-loaded cylindrical guiding structures (circular waveguide and coaxial lines) where the normalized phase characteristics and distribution of field components were found. Also, a couple of azimuthally magnetized cases of a ferrite rod placed at the center of a circular 


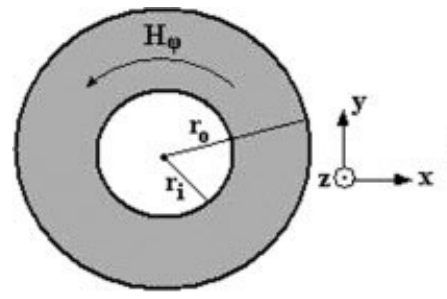

(a)

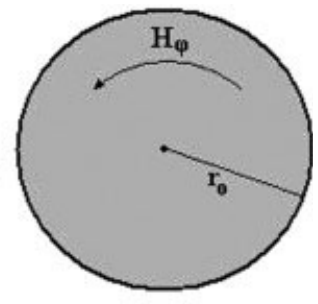

(b)
Figure 1. Azimuthally magnetized ferrite-loaded circular guiding structures. (a) Completely filled coaxial line. (b) Completely filled circular waveguide.

waveguide partially filling the cross section were discussed in [11]. In [12], the variational method was used to find the normalized propagation constant $\beta / k_{0}$ for an azimuthally magnetized ferrite-filled circular waveguide. For longitudinal magnetization, the external static field will be along the $z$-axis. Different researchers studied and solved for the propagation characteristics of ferrite-loaded circular structures as in [13-16]. In [17], Dib and Omar presented a full wave dispersion analysis of multilayer cylindrical transmission lines containing (azimuthally and longitudinally) magnetized ferrite material using 2DFDTD method and spectral domain analysis (SDA). The electromagnetic wave propagation in transversely magnetized ferrite filled circular waveguide was completely analyzed in cylindrical coordinates in [18]. TE and TM modes and a study of the cutoff characteristics for both the radial and azimuthally magnetized ferrite-loaded circular waveguides were presented in [19]. In [20-23], phase shifter was produced as one application for ferrite. A recent research for implementation of left handed materials using ferrite loaded waveguides has been presented in [2426].

In this article, the FDFD method is used to analyze azimuthally magnetized ferrite-loaded circular waveguide. Emphasis is placed on the $\mathrm{TE}_{0 \mathrm{~m}}$ modes that are rotationally symmetric. This reduces our problem to a one-dimensional (1D) problem, and thus, only the 1DFDFD method is presented here. Starting from Maxwell's equations, a full-wave analysis is presented for coaxial lines and circular waveguides completely filled with azimuthally magnetized ferrites. The results that are obtained after solving these FDFD equations come through solving the eigen-value problem, where the obtained eigen-values and eigen-vectors are used to produce the propagation constants and the distribution of the fields. The obtained results are compared with those obtained using other techniques, and a very good agreement is obtained.

\section{ANALYSIS}

The cross sections of a coaxial line and circular waveguide completely filled with azimuthally magnetized ferrites are shown in Figure 1. The FDFD method is formulated below to analyze these circular structures to find the normalized propagation constant $\beta / k_{0}$ and the electric and magnetic fields distributions at a given frequency.

Assuming propagation in $+z$-direction, all field components will have a longitudinal variation of the form $e^{-j \beta z}$ where $\beta$ is the phase constant in $(\mathrm{rad} / \mathrm{m})$. For the rotationally symmetric $\mathrm{TE}_{0 \mathrm{~m}}$ modes, only $E_{\varphi}$, $H_{\rho}$ and $H_{z}$ exist and can be written as:

$$
\begin{gathered}
\bar{E}(\rho, \varphi, z)=\left[E_{\varphi}(\rho) \hat{\varphi}\right] e^{-j \beta z} \\
\bar{H}(\rho, \varphi, z)=\left[H_{\rho}(\rho) \hat{\rho}+H_{z}(\rho) \hat{z}\right] e^{-j \beta z}
\end{gathered}
$$

By considering normalized fields: $\bar{H}^{\prime}=\bar{H} \times \sqrt{\eta_{0}}$ and $\bar{E}^{\prime}=\bar{E} \times \sqrt{\eta_{0}}$, where $\eta_{0}=\sqrt{\mu_{0} / \varepsilon_{0}}$ is the freespace intrinsic impedance, then, modified Maxwell's equations can be written as (the normalized mark "/" will be omitted):

$$
\begin{gathered}
\nabla \times \bar{E}=-j k_{0}\left[\mu_{\mathrm{r}}\right] \bar{H} \\
\nabla \times \bar{H}=j k_{0}\left[\varepsilon_{\mathrm{r}}\right] \bar{E}
\end{gathered}
$$

where: $k_{0}=\omega \sqrt{\mu_{0} \varepsilon_{0}}, \omega$ is the input angular frequency of the electromagnetic wave, $\left[\varepsilon_{\mathrm{r}}\right]$ is the dielectric constant tensor, which is assumed to be diagonal as:

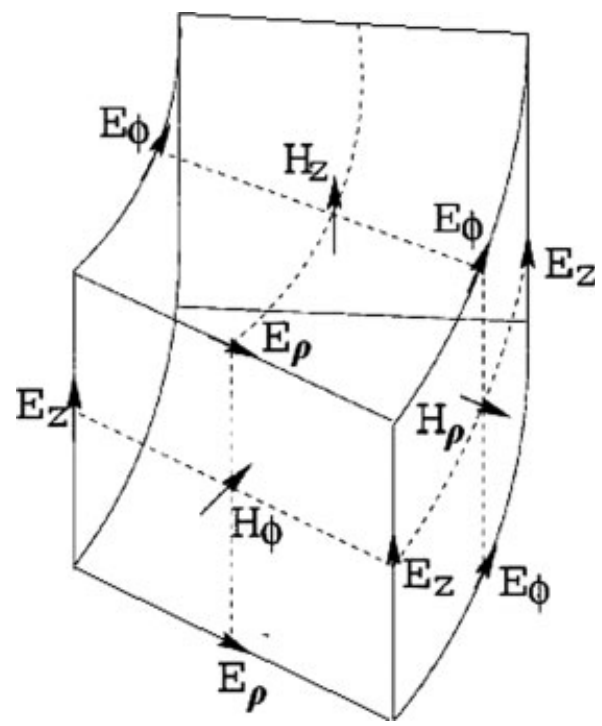

Figure 2. Conventional Yee's 3-D grid for circular structures. 
$[\varepsilon]=\varepsilon_{0}\left[\varepsilon_{\mathrm{r}}\right]=\left[\begin{array}{ccc}\varepsilon_{\rho \rho} & 0 & 0 \\ 0 & \varepsilon_{\varphi \varphi} & 0 \\ 0 & 0 & \varepsilon_{z z}\end{array}\right]=\varepsilon_{0}\left[\begin{array}{ccc}\varepsilon_{\mathrm{r} \rho} & 0 & 0 \\ 0 & \varepsilon_{\mathrm{r} \varphi} & 0 \\ 0 & 0 & \varepsilon_{\mathrm{r} z}\end{array}\right]$

Ferrite regions are characterized by the Polder permeability tensor $[\mu]$ that is related to the direction of the external static magnetic field. In the case of the azimuthally magnetization, $\left[\mu_{\mathrm{r}}\right]$ can be written as:

$$
\begin{array}{r}
{[\mu]=\mu_{0}\left[\mu_{\mathrm{r}}\right]=\mu_{0}\left[\begin{array}{ccc}
1 & 0 & -j \alpha \\
0 & 1 & 0 \\
j \alpha & 0 & 1
\end{array}\right]} \\
=\mu_{0}\left[\begin{array}{ccc}
\mu_{\mathrm{r} 11} & 0 & \mu_{\mathrm{r} 13} \\
0 & 1 & 0 \\
\mu_{\mathrm{r} 31} & 0 & \mu_{\mathrm{r} 33}
\end{array}\right]
\end{array}
$$

where, $\alpha=\gamma M_{\mathrm{s}} / \omega ; M_{\mathrm{s}}$ is the (DC) saturation magnetization (in $\mathrm{A} / \mathrm{m})$ and $\gamma=-2.21042 \times 10^{5}(\mathrm{rad} / \mathrm{s})$ $(\mathrm{A} / \mathrm{m})$ is the gyromagnetic ratio.

Figure 2 shows the conventional 3D cylindrical Yee's cell; while Figure 3 shows the compact 1D grid that will be used here to discretize Maxwell's equations.

According to the compact 1D grid in Figure 3, and starting from Maxwell's equations in (3) and (4) and substituting for both $\left[\varepsilon_{\mathrm{r}}\right]$ and $\left[\mu_{\mathrm{r}}\right]$, and using interpolation to avoid the problem of coupling between $H_{\rho}$ and $H_{z}$ that results from applying the static magnetic field, 1D-FDFD equations can be derived [27], and are given as follows:

$$
\frac{\beta}{k_{0}} E_{\varphi}(n)=-\mu_{\mathrm{r} 11} H_{\rho}(n)-\frac{\mu_{\mathrm{r} 13}}{2} H_{z}(n)-\frac{\mu_{\mathrm{r} 13}}{2} H_{z}(n+1)
$$

$$
\frac{\beta}{k_{0}} H_{\rho}(n)=\frac{1}{j k_{0} h_{\rho}} H_{z}(n)-\frac{1}{j k_{0} h_{\rho}} H_{z}(n+1)-\varepsilon_{\mathrm{r} \varphi} E_{\varphi}(n)
$$

$$
\begin{aligned}
& \frac{\beta}{k_{0}} H_{z}(n)=\frac{\mu_{\mathrm{r} 11} n}{j k_{0} \mu_{\mathrm{r} 33}(n-.5) h_{\rho}} H_{\rho}(n) \\
& -\frac{\mu_{\mathrm{r} 11}(n-1)}{j k_{0} \mu_{\mathrm{r} 33}(n-.5) h_{\rho}} H_{\rho}(n-1) \\
& +\left[\frac{\mu_{\mathrm{r} 13} n}{j 2 k_{0} \mu_{\mathrm{r} 33}(n-.5) h_{\rho}}-\frac{\mu_{\mathrm{r} 13}(n-1)}{j 2 k_{0} \mu_{\mathrm{r} 33}(n-.5) h_{\rho}}\right] H_{z}(n) \\
& +\left[-\frac{\mu_{\mathrm{r} 13}(n-1)}{j 2 k_{0} \mu_{\mathrm{r} 33}(n-.5) h_{\rho}}-\frac{\mu_{\mathrm{r} 31}}{j 2 \mu_{\mathrm{r} 33} k_{0} h_{\rho}}\right] H_{z}(n-1) \\
& +\left[\frac{\mu_{\mathrm{r} 13} n}{j 2 k_{0} \mu_{\mathrm{r} 33}(n-.5) h_{\rho}}+\frac{\mu_{\mathrm{r} 31}}{j 2 \mu_{\mathrm{r} 33} k_{0} h_{\rho}}\right] H_{z}(n+1) \\
& \quad+\frac{\mu_{\mathrm{r} 31} \varepsilon_{\mathrm{r} \varphi}}{2 \mu_{\mathrm{r} 33}} E_{\varphi}(n)+\frac{\mu_{\mathrm{r} 31} \varepsilon_{\mathrm{r} \varphi}}{2 \mu_{\mathrm{r} 33}} E_{\varphi}(n-1)
\end{aligned}
$$

These FDFD equations can be applied directly to solve for the central part of the circular guiding structures that are completely filled with ferrite away from the conductor boundaries. On the conductor boundaries, modified FDFD equations have to be derived according to the following boundary conditions:

$$
\begin{gathered}
B_{\text {normal }}=0 \text {; i.e. } B_{\rho}=0 . \\
E_{\text {tangential }}=0 \text {; i.e. } E_{z}, E_{\varphi}=0
\end{gathered}
$$

where $B$ is the magnetic flux density. Applying these boundary conditions transforms the derivation of the modified FDFD equations to a complex level because of the coupling between $H_{\rho}$ and $H_{z}$. New four FDFD equations, according to the position and the distance from both the outer and inner conductors (in the case of the completely filled coaxial line), have to be derived. At the outer conductor boundary and substituting for $B_{\rho}$ in (10), extrapolation has to be used to evaluate $H_{z}$ at the conducting boundary, which gives the following equation:

$$
B_{\rho}(n)=\mu_{\mathrm{r} 11} H_{\rho}(n)+\frac{\mu_{\mathrm{r} 13}}{2}\left[3 H_{z}(n)-H_{z}(n-1)\right]=0
$$

Now, a modified FDFD equation can be derived to solve for the normal magnetic field $H_{\rho}$ at the outer conductor boundary as follows:

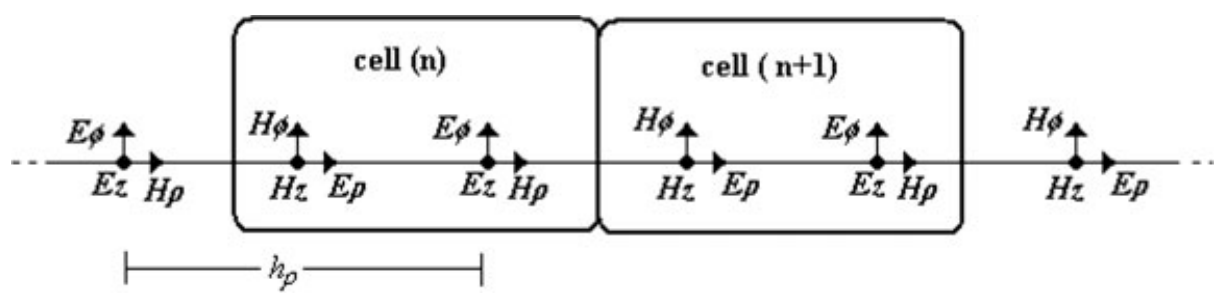

Figure 3. Compact 1D grid. 


$$
\begin{aligned}
& \frac{\beta}{k_{0}} H_{\rho}(n)=\left[\frac{3 \mu_{\mathrm{r} 13} \mu_{\mathrm{r} 11}(n-1)}{2 j \mu r_{11} \mu_{\mathrm{r} 33} h_{\rho} k_{0}(n-.5)\left(1-\frac{3 \mu_{\mathrm{r} 1} \mu_{\mathrm{r} 31}}{4 \mu_{\mathrm{r} 11} \mu_{\mathrm{r} 33}}\right)}+\frac{\mu_{\mathrm{r} 13} \mu_{\mathrm{r} 11}(n-1)}{2 j \mu_{\mathrm{r} 11} \mu_{\mathrm{r} 33} h_{\rho} k_{0}(n-1.5)\left(1-\frac{3 \mu_{\mathrm{r} 13} \mu_{\mathrm{r} 31}}{4 \mu_{\mathrm{r} 11} \mu_{\mathrm{r} 33}}\right)}\right] H_{\rho}(n-1) \\
& -\frac{\mu_{\mathrm{r} 13} \mu_{\mathrm{r} 11}(n-2)}{2 j \mu_{\mathrm{r} 11} \mu_{\mathrm{r} 33} h \rho k_{0}(n-1.5)\left(1-\frac{3 \mu_{\mathrm{r} 13} \mu_{\mathrm{r} 13}}{4 \mu_{\mathrm{r} 11} \mu_{\mathrm{r} 33}}\right)} H_{\rho}(n-2) \\
& +\left[-\frac{3 \mu_{\mathrm{r} 13} \mu_{\mathrm{r} 31} \varepsilon_{\mathrm{r} \varphi}}{4 \mu_{\mathrm{r} 11} \mu_{\mathrm{r} 33}\left(1-\frac{3 \mu_{\mathrm{r} 13} \mu_{\mathrm{r} 31}}{4 \mu_{\mathrm{r} 11} \mu_{\mathrm{r} 33}}\right)}+\frac{\mu_{\mathrm{r} 13} \mu_{\mathrm{r} 31} \varepsilon_{\mathrm{r} \varphi}}{4 \mu_{\mathrm{r} 11} \mu_{\mathrm{r} 33}\left(1-\frac{3 \mu_{\mathrm{r} 13} \mu_{\mathrm{r} 31}}{4 \mu_{\mathrm{r} 11} \mu_{\mathrm{r} 3}}\right)}\right] E_{\varphi}(n-1) \\
& +\frac{\mu_{\mathrm{r} 13} \mu_{\mathrm{r} 31} \varepsilon_{\mathrm{r} \varphi}}{4 \mu_{\mathrm{r} 11} \mu_{\mathrm{r} 33}\left(1-\frac{3 \mu_{\mathrm{r} 1} \mu_{\mathrm{r} 31}}{4 \mu_{\mathrm{r} 11} \mu_{\mathrm{r} 33}}\right)} E_{\varphi}(n-2) \\
& +\left[\begin{array}{l}
+\frac{3 \mu_{\mathrm{r} 13} \mu_{\mathrm{r} 13}(n-1)}{4 j \mu_{\mathrm{r} 11} \mu_{\mathrm{r} 33} h_{\rho} k_{0}(n-.5)\left(1-\frac{3 \mu_{\mathrm{r} 13} \mu_{\mathrm{r} 31}}{4 \mu_{\mathrm{r} 11} \mu_{\mathrm{r} 33}}\right)}+\frac{3 \mu_{\mathrm{r} 13} \mu_{\mathrm{r} 31}}{4 j \mu_{\mathrm{r} 11} \mu_{\mathrm{r} 33} h_{\rho} k_{0}\left(1-\frac{3 \mu_{\mathrm{r} 13} \mu_{\mathrm{r} 31}}{4 \mu_{\mathrm{r} 11} \mu_{\mathrm{r} 33}}\right)} \\
+\frac{\mu_{\mathrm{r} 13} \mu_{\mathrm{r} 13}}{4 j \mu_{\mathrm{r} 11} \mu_{\mathrm{r} 33} h_{\rho} k_{0}(n-1.5)\left(1-\frac{3 \mu_{\mathrm{r} 1} \mu_{\mathrm{r} 31}}{4 \mu_{\mathrm{r} 11} \mu_{\mathrm{r} 33}}\right)}
\end{array}\right] H_{z}(n-1) \\
& +\left[\begin{array}{c}
+\frac{3 \mu_{\mathrm{r} 13} \mu_{\mathrm{r} 13}(n-1)}{4 j \mu_{\mathrm{r} 11} \mu_{\mathrm{r} 33} h_{\rho} k_{0}(n-.5)\left(1-\frac{3 \mu_{\mathrm{r} 13} \mu_{\mathrm{r} 13}}{4 \mu_{\mathrm{r} 1} \mu_{\mathrm{r} 33}}\right)}-\frac{3 \mu_{\mathrm{r} 13} \mu_{\mathrm{r} 31}}{4 j \mu_{\mathrm{r} 11} \mu_{\mathrm{r} 33} h_{\rho} k_{0}\left(1-\frac{3 \mu_{\mathrm{r} 13} \mu_{\mathrm{r} 31}}{4 \mu_{\mathrm{r} 1} \mu_{\mathrm{r} 33}}\right)} \\
+\frac{\mu_{\mathrm{r} 13} \mu_{\mathrm{r} 13}(n-1)}{4 j \mu_{\mathrm{r} 11} \mu_{\mathrm{r} 33} h_{\rho} k_{0}(n-1.5)\left(1-\frac{3 \mu_{\mathrm{r} 13} \mu_{\mathrm{r} 31}}{4 \mu_{\mathrm{r} 1} \mu_{\mathrm{r} 33}}\right)}+\frac{\mu_{\mathrm{r} 13} \mu_{\mathrm{r} 31}}{4 j \mu_{\mathrm{r} 11} \mu_{\mathrm{r} 33} h_{\rho} k_{0}\left(1-\frac{3 \mu_{\mathrm{r} 13} \mu_{\mathrm{r} 31}}{4 \mu_{\mathrm{r} 11} \mu_{\mathrm{r} 33}}\right)}
\end{array}\right] H_{z}(n) \\
& +\left[-\frac{\mu_{\mathrm{r} 13} \mu_{\mathrm{r} 13}(n-2)}{4 j \mu_{\mathrm{r} 11} \mu_{\mathrm{r} 33} h_{\rho} k_{0}(n-1.5)\left(1-\frac{3 \mu_{\mathrm{r} 13} \mu_{\mathrm{r} 31}}{4 \mu_{\mathrm{r} 11} \mu_{\mathrm{r} 33}}\right)}-\frac{\mu_{\mathrm{r} 13} \mu_{\mathrm{r} 31}}{4 j \mu_{\mathrm{r} 11} \mu_{\mathrm{r} 33} h_{\rho} k_{0}\left(1-\frac{3 \mu_{\mathrm{r} 13} \mu_{\mathrm{r} 31}}{4 \mu_{\mathrm{r} 11} \mu_{\mathrm{r} 33}}\right)}\right] H_{z}(n-2)
\end{aligned}
$$

The same procedure is applied at the surface of the inner conductor (in the case of coaxial line), where extrapolation is also used as follows:

$$
\begin{aligned}
& B_{\rho}(n)=\mu_{\mathrm{r} 11} H_{\rho}(n)+\frac{\mu_{\mathrm{r} 13}}{2}[ 3 H_{z}(n+1) \\
&\left.-H_{z}(n+2)\right]=0
\end{aligned}
$$

The values for the longitudinal magnetic field component that are near the outer and inner conductors are modified too because these field components are affected by the neighboring modification on the radial normal magnetic fields. Using (12) again to derive $H_{z}$ at the outer conductor boundary with respect to all boundary conditions and using (13) to substitute for $H_{\rho}$ give a new $H_{z}$ FDFD equation at this outer conductor [27].

Finally, a complete system of 1D-FDFD equations for both the ferrite and conductors parts of the circular guiding structures is ready to be solved. The FDFD equations are written in the form of an eigenvalue problem as follows:

$$
A \bar{X}=\frac{\beta}{k_{0}} \bar{X}
$$

where the coefficient matrix $A$ is the right hand sparse matrix coefficients taken from the FDFD equations, $\bar{X}$ $=\left\{H_{\rho}, E_{\varphi}, H_{z}\right\}^{T}$ is the field vector, and $\beta / k_{0}$ are the normalized eigen-values which are found by solving:

$$
\operatorname{det}\left\{[A]-\frac{\beta}{k_{0}}[I]\right\}=0
$$

where $[I]$ is the identity matrix.

\section{NUMERICAL RESULTS}

To verify the proposed 1D-FDFD techniques, two completely filled circular guiding structures with an outer conductor radius of $5 \mathrm{~mm}$ filled with an azimuthally magnetized ferrite with $\varepsilon_{\mathrm{r}}=12.5$ are analyzed. To compare the FDFD results with those presented in $[9,10]$, normalized values for both $f$ and $\beta$ defined as $f_{\mathrm{n}}=2 \pi r_{0} f \sqrt{\mu_{0} \varepsilon_{0} \varepsilon_{\mathrm{r}}}$ and $\beta_{\mathrm{n}}=\beta /\left(2 \pi f \sqrt{\varepsilon_{\mathrm{r}}}\right)$ are used. Figures 4-6 show some sample results for the dispersion characteristics of azimuthally magnetized ferrite-filled coaxial line. FDFD results are compared with those presented in $[9,10]$, and an excellent agreement is obtained. 


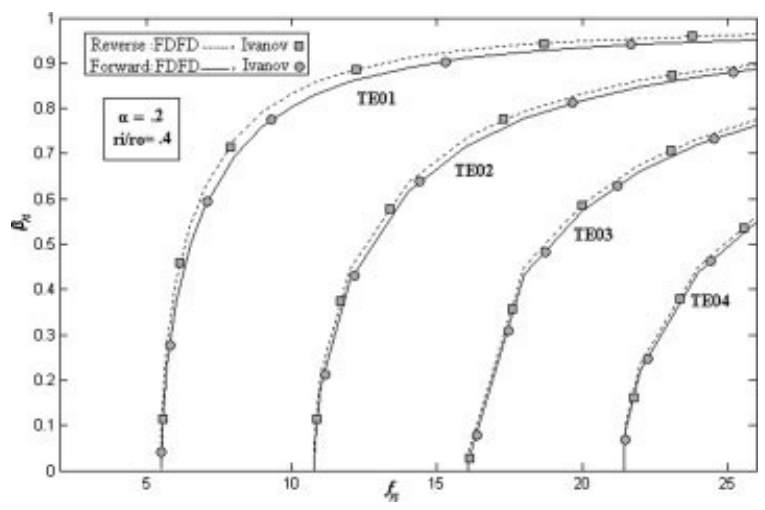

Figure 4. Dispersion characteristics of azimuthally magnetized ferrite filled coaxial line with $\alpha=0.2$, and ri/ro $=$ 0.4 .

As seen in Figures 4-6, there are two possible values for $\beta_{n}$; forward phase constant $\beta_{n}^{+}$and reverse phase constant $\beta_{n}^{-}$. This shows that this structure is nonreciprocal because of the existence of the ferrite material. It is clear that increasing $\alpha$ increases the cutoff frequency for the same mode even though the ratio between the inner and the outer conductors' radii is kept constant. Moreover, the difference between $\beta_{n}^{+}$and $\beta_{n}^{-}$increases as $\alpha$ increases.

The same 1D-FDFD algorithm is used to analyze circular waveguides completely filled with ferrite. Figure 7 shows a sample result. The nonreciprocity behavior is very clear in Figure 7. It can be also seen that the circular waveguide could work within a small range of frequencies below the cutoff of the forward wave. We can see that for the reverse wave, two different values for $\left(\beta_{n}^{-}\right)$appear below the cutoff frequency of the forward wave, which means that in this range, the waveguide could transmit the reverse wave, and isolate the forward one. But, by increasing

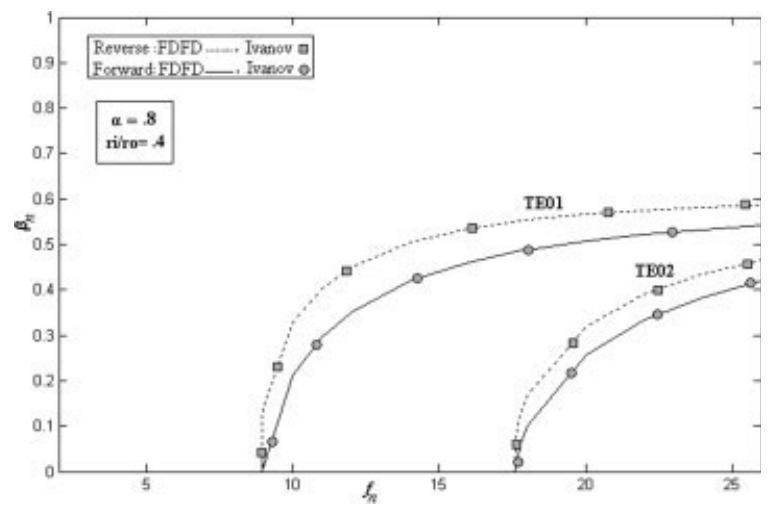

Figure 5. Dispersion characteristics of azimuthally magnetized ferrite filled coaxial line with $\alpha=0.8$, and ri/ro $=$ 0.4 .

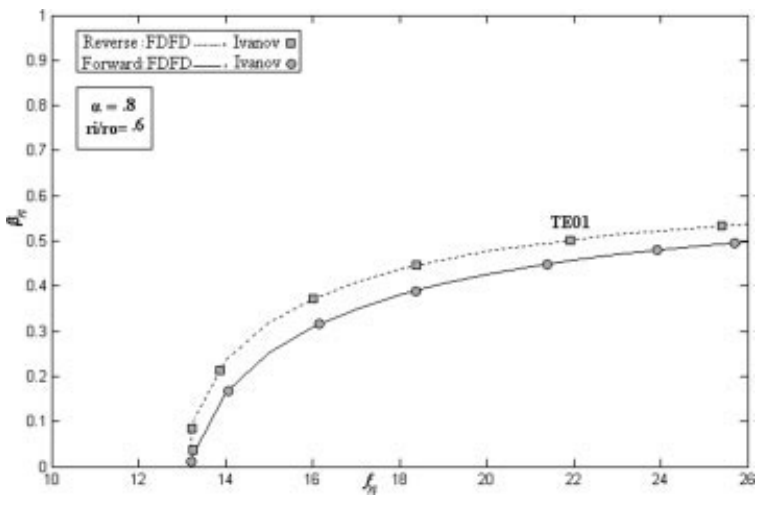

Figure 6. Dispersion characteristics of azimuthally magnetized ferrite filled coaxial line with $\alpha=0.8$, and ri/ro $=$ 0.6 .

the frequency, one of these two values becomes zero, and the other one keeps increasing as the frequency is increased. The forward wave behaves normally as it has a cutoff frequency below which it becomes evanescent, and above which it becomes a propagating wave.

By reversing the sign of $\alpha$, magnetization will reverse its direction (from $+\varphi$ to $-\varphi$ ), which means that there will be a phase shift of a value $\Delta \beta$ because of changing the direction of magnetization. The value of $\Delta \beta$ can be evaluated at any of these $\beta$ 's and will bring the circular waveguide from the transmitting state $\beta_{n}^{-} \neq 0$ to the cutoff state $\beta_{n}^{+}=0$. This means that, the microwave signal which was propagating along the reverse direction for $(\alpha=+0.95)$ becomes in the cutoff state after reversing the magnetization direction $(\alpha=-0.95)$. This rather strange behavior of the azimuthally magnetized ferrite-filled circular waveguides gives us another application for these structures which is the effective cutoff isolators.

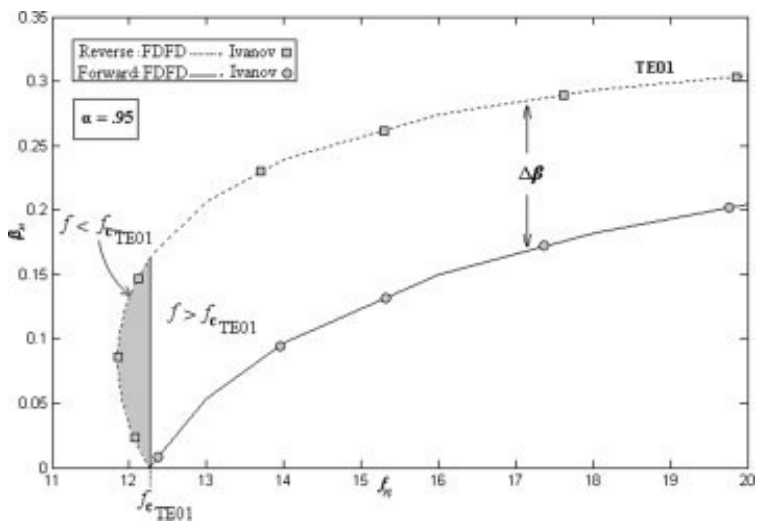

Figure 7. Dispersion characteristics of azimuthally magnetized ferrite filled circular waveguide. 


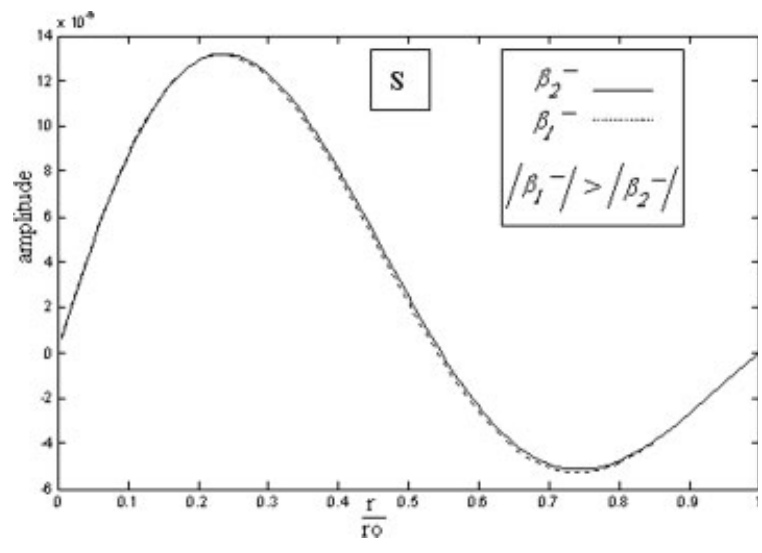

Figure 8. Poynting vector for reverse waves when $\alpha=$ 0.95 and $f_{n}=11.854$.

To give more details about propagation below the forward wave cutoff, Poynting vectors for $\alpha=0.95$ will be plotted at different values of $f_{n}$. At a value of $f_{n}=11.854<f_{\text {cTE 01 }}$, two negative values of $\beta ; \beta_{1}^{-}$ and $\beta_{2}^{-}$appear. The Poynting vector corresponding to these two negative phase constants is shown in Figure 8 . On the other hand, for $f_{n}=20>f_{\text {cTE } 01}, \beta^{+}$ and $\beta^{-}$appear. The Poynting vector corresponding to these two phase constants is shown in Figure 9.

According to Figure 8, it is clear that azimuthally magnetized ferrite filled circular waveguide supports backward waves for frequencies $f<f_{\text {cTE } 01}$, which are in the bulge region shown clearly in Figure 7. In this region and according to the given values of $\alpha$; $\int_{\mathrm{r}_{\mathrm{i}} / \mathrm{r}_{0}}^{1} S^{-} d \rho>0$, even though we have negative values of the propagation constants: $\beta_{1}^{-}$and $\beta_{2}^{-}$, that is negative values for the phase velocity. This means that the phase velocity has direction which is opposite to the direction of the Poynting vector, which gives what is known as backward wave. For the range of frequencies $\left(f>f_{\text {cTE } 01}\right)$, as in Figure 9, ferrite filled circular waveguide returns to support the normal waves since: $\int_{\mathrm{r}_{\mathrm{i}} / \mathrm{r}_{0}}^{1} S^{-} d \rho<0$ for $\beta^{-} ;$and $\int_{\mathrm{r}_{\mathrm{i}} / \mathrm{r}_{0}}^{1} S^{+} d \rho>0$ for $\beta^{+}$.

From the numerical values of $\Delta \beta$ in the previous examples, simple formulas as in [28] can be derived based on the values of $\alpha$ and $r_{n}$, where $r_{n}=2 r_{0} \pi f \sqrt{\varepsilon_{\mathrm{r}}}$ is the normalized guide radius. $\Delta \beta$ can be found for any waveguide filled with ferrite at any value of frequency and magnetization and/or radius as:

$$
\begin{gathered}
\Delta \beta=A|\alpha| \\
\Delta \beta=B / r_{\mathrm{n}} \\
\Delta \beta=C|\alpha| / r_{\mathrm{n}}
\end{gathered}
$$

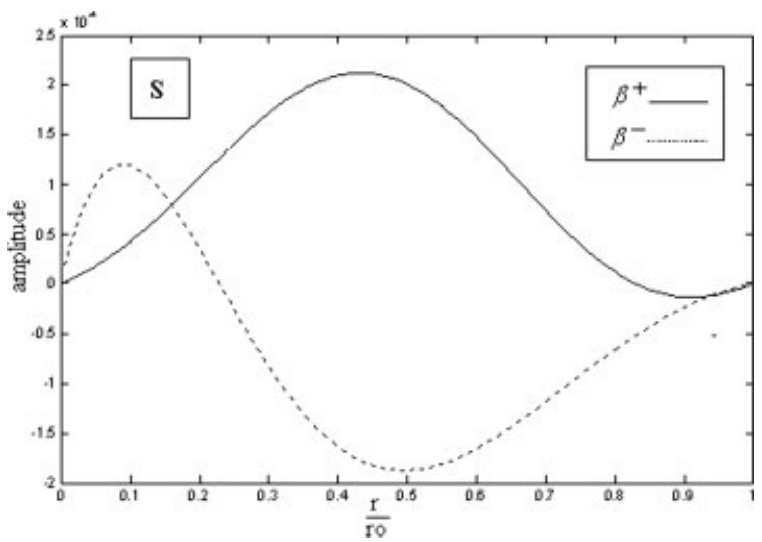

Figure 9. Poynting vector when $\alpha=0.95$ and $f_{n}=20$.

In the above equations, $A, B$, and $C$ are practically constant values at fixed values of $r_{n}$ and $\alpha$ in (17) and (18), respectively, or different values for these parameters as in (19). As an example of using eqs. (17)-(19) to determine $\Delta \beta$, Figure 10 shows the way of finding the constant $A$ from (17).

From (17), it is clear that $A$ is practically constant for different values of $\alpha$ at fixed $r_{n}$, which means that this curve represents the approximation curve for all the values of $A(\alpha)$. As seen from (17) and (19) and Figure 10 that the constant $C$ can be found directly by multiplying any value of $A$ with its corresponding $r_{n}$, which means the area of any arbitrary rectangle: abcd, afgh, or aejk which is a constant value as have been proposed for this scheme in [28]. The results in Figure 10 state that propagation is possible for the values of $\alpha$ that are larger than the given ones, which gives us an indication of the cutoff state of this waveguide.

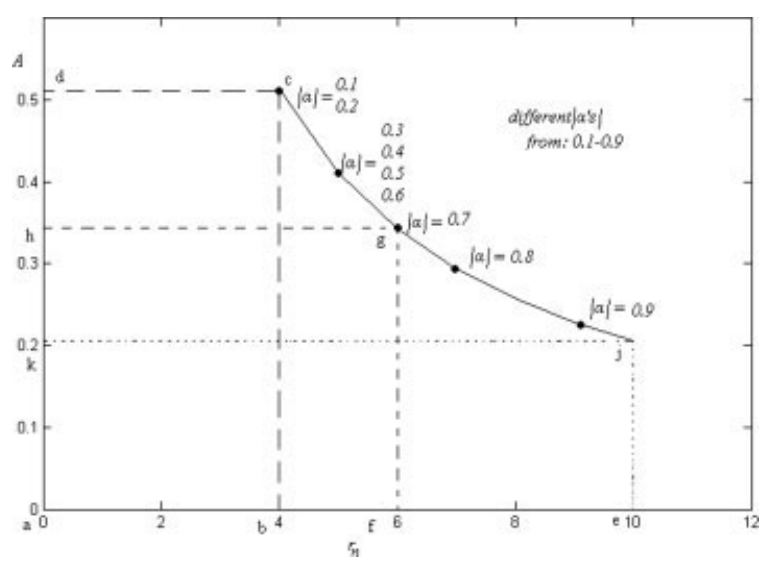

Figure 10. The factor $A$ versus $r_{n}$ at range of $\alpha$ 's $=$ $(0.1-0.9)$. 


\section{CONCLUSIONS}

FDFD method was formulated to solve for the dispersion characteristics of azimuthally magnetized ferritefilled circular guiding structures. Several values of magnetization $(\alpha)$ were taken into our simulations. It was observed that increasing $\alpha$ will increase the magnetic anisotropy, and as a result, it will increase the difference between $\beta^{+}$and $\beta^{-}$. The most important property was the possibility of the backward wave propagation at certain frequencies below the cutoff. Several dispersion characteristics were presented for the rotationally symmetric $\mathrm{TE}_{0 \mathrm{~m}}$ modes. These modes play an important role in most applications for the circular structures that are magnetized with an azimuthally directed static field. Deriving the formulas for $\Delta \beta$ enables us to calculate $\Delta \beta$ at any given frequency based on the normalized guide radius or the value of $\alpha$. Unlike the analytical technique used in $[9,28]$, the presented FDFD method can be easily extended to analyze waveguides that are filled with multi-layers of ferrite and isotropic media. Moreover, it can be extended to 2D-FDFD, which can be used to study other circular cylindrical guiding structures containing ferrite media, for example, cylindrical microstrip line with a ferrite substrate [17].

\section{REFERENCES}

1. C.M. Arft and A. Knoesen, An efficient finite-difference frequency-domain method for waveguideing structures including thin curved layers, Microwave Opt Technol Lett 48 (2006), 453-457.

2. Q. Zhang, X. Zhou, and H. Yi, propagation characteristic analysis of ridged circular waveguide using $2 \mathrm{D}$ finite-difference frequency-domain method, Int $\mathrm{J}$ Microwave RF CAE 15 (2005), 264-271.

3. J.-X. Niu, Q. Zhang, X.-L. Zhou, and Z.-Y. Shan, A compact 2-D finite-difference frequency-domain method for dispersion characteristics analysis of trapezoidal-ridge waveguides, Int $\mathrm{J}$ Infrared Millimet Waves 29 (2008), 519-526.

4. M. Paradavi-Horvath, Microwave applications of soft ferrites, J Magn Magn Mater 215-216 (2000), 171-183.

5. A.J. Baden Fuller, An introduction to the use of ferrite and garnet materials at microwave and optical frequencies, Electron Commun Eng J 7 (1995), 72-80.

6. D.M. Pozar, Microwave engineering, 2nd ed., Wiley, New York, 1998.

7. B. Lax and K. Button, Microwave ferrites and ferrimagnetics, 1st ed., McGraw Hill, New York, 1962.

8. E. Jerby, A. Aharony, and G. Breitmeier, Ferrite guided resonance maser, Phys Rev E 65 (2002), 2.

9. K.P. Ivanov, Propagation along azimuthally magnetized, ferrite-loaded circular guide, Radio Sci 19 (1984), 1305-1310.
10. K.P. Ivanov and R. Pregla, Analysis of circular waveguides with azimuthally magnetized ferrite, 27th Eu. Microwave Conf. (1997), 229-231.

11. M. Masuda, N.S. Chang, and Y. Matsuo, Azimuthally dependent magnetostatic modes in the cylindrical ferrites, IEEE Trans Microwave Theory Tech 19 (1971), 834-836.

12. I.V. Lindell, Variational methods for nonstandard eigenvalue problems in waveguide and resonator analysis, IEEE Trans Microwave Theory Tech 30 (1982), 1194-1204.

13. J. Helszajn and A.A.P. Gibson, Mode nomenclature of circular gyromaganetic and anisotropic waveguides with magnetic and open walls, IEE Proc 134 (1987), 488-496.

14. M. Kales, Modes in waveguides containing ferrites, J Appl Phys 24 (1953), 604-608.

15. G.C. Chinn, L.W. Epp, and G.M. Wilkins, Determinations of the eigenfrequencies of a ferrite filled cylindrical cavity resonator using the finite element method, IEEE Trans Microwave Theory Tech 43 (1995), 1207-1209.

16. S.S. Sandler, An approximate solution to some ferrite filled waveguide problems with longitudinal magnetization, IRE Trans Microwave Theory Tech 9 (1961), $162-168$.

17. N. Dib and A. Omar, Dispersion analysis of multilayer cylindrical transmission lines containing magnetized ferrite substrates, IEEE Trans Microwave Theory Tech 50 (2002), 1730-1736.

18. A. Konstantinov and V. Kalesinskas, EM wave propagation in ferrite-filled transversely magnetized circular waveguide, Int J Infrared Millimet Waves 21 (2000), 1869-1878.

19. W. Che and E.K. Yung, Cutoff characteristic of modes in a circular waveguide filled with microwave ferrites, J Electromagn Waves Appl 16 (2002), 11031118.

20. C.R. Boyd Jr., Microwave phase shift using ferritefilled waveguide below cutoff, IEEE Trans Microwave Theory Tech 45 (1997), 2402-2407.

21. D.M. Bolle and G.S. Heller, Theoretical considerations on the use of circularly symmetric te modes for digital ferrite phase shifters, IEEE Trans Microwave Theory Tech 13 (1965), 421-426.

22. G.N. Tsandoulas and W.J. Ince, Modal inversion in circular waveguide-part I: Theory and phenomenology, IEEE Trans Microwave Theory Tech 19 (1971), 386-392.

23. G.N. Tsandoulas and W.J. Ince, Modal inversion in circular waveguide-part II: Application to latching nonreciprocal phasers, IEEE Trans Microwave Theory Tech 19 (1971), 393-400.

24. S. Antipov, L.K. Spentzouris, M. Conde, W. Gai, W. Liu, R. Konecny, J.G. Power, and Z. Yusof, Metamaterial loaded waveguides for acceleratore applications. IEEE Particle Accelerator Conference, June 2007, pp. 2906-2908. 
25. T. Ueda and M. Tsutsumi, Left handed transmission characteristics of rectangular waveguides periodically loaded with ferrite, IEEE Trans Microwave Theory Tech 41 (2005), 3532-3537.

26. A. Decant and M. Okoniewski, Broadband double negative material from ferrite loaded metallic waveguides, Electron Lett 42 (2006), 4-5.

27. M.R. Rawashdeh, Full-wave analysis of circular guiding structures using the two-dimensional finite differ- ence frequency domain method, M.Sc. Thesis, Department of Electrical Engineering, Jordan University of Science and Technology, Irbid, Jordan, 2008.

28. G.N. Georgiev and M.N. Georgieva-Grosse, Formulae for differential phase shift computation in an azimuthally magnetized circular ferrite waveguide, In Proc Millenn Conf Antennas Propagat AP-2000, Davos, Switzerland, April 9-14, 2000, Abstracts, Vol. IAntennas, p. 517, in CD, paper 1002.

\section{BIOGRAPHIES}

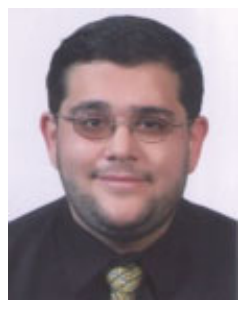

Mohammad R. Rawashdeh was born in 1981. He obtained his B.Sc. (2004) and M.Sc. (2008) in electrical engineering, from Jordan University of Science and Technology (JUST), Jordan. During 20042005, Rawashdeh worked as a communication engineer in Jordan Telecom Group/ Orange. He is now a lecturer in the electrical engineering department at JUST. His research interests are in computational electromagnetics, applied mathematics, and applied physics.

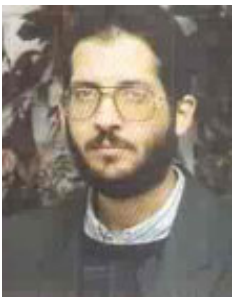

Nihad Dib obtained his B.Sc. and M.Sc. in EE from Kuwait University in 1985 and 1987, respectively. He obtained his Ph.D. in EE (major in Electromagnetics and Microwaves) in 1992 from University of Michigan, Ann Arbor. Then, he worked as an assistant research scientist in the radiation laboratory at the same school. In September 1995, he joined the EE department at Jordan University of Science and Technology (JUST) as an assistant professor, and became a full professor in August 2006. His research interests are in computational electromagnetics and modeling of planar circuits. 